International Mathematical Forum, 2, 2007, no. 68, 3357 - 3369

\title{
A Combinatorial Game Mathematical Strategy Planning Procedure for a Class of Chess Endgames
}

\author{
Zvi Retchkiman Königsberg \\ Instituto Politécnico Nacional, CIC \\ Mineria 17-2, Col. Escandon, Mexico D.F 11800, Mexico \\ mzvi@cic.ipn.mx
}

\begin{abstract}
In this paper a strategy planning procedure for a class of chess endgames is given. The class of chess endgames considered are those chess games that are built around a Zugzwang, are just formed by pawns and, whose configuration can be decomposed as a sum of independent subgames restricted to belong to one of the following cases: i) Zero or fuzzy games, and ii) Positive or negative games where the winner has the possibility of driving the game to case (i). In addition some tempos are allowed. The main contribution consists in giving a strategy plan procedure of how a chess player checkmates or draws knowing that he is in a winning position using combinatorial game mathematical tools. By a winning position in a game it is understood a position where one of the players makes the last move in that game and as a consequence forces his opponent to start playing in the next game and so on, until at the end plays first in the Zugzwang and draws or loses the chess game.
\end{abstract}

Mathematics Subject Classification: 91A46, 93C95

Keywords: Combinatorial game theory, Chess endgames, Chess modeling

\section{Introduction}

The paper presents a strategy planning procedure for a class of chess endgames. The class of chess endgames considered are those chess games that are built around a Zugzwang position ${ }^{1}$, are just formed by pawns and, whose configuration can be decomposed as a sum of independent subgames restricted to

\footnotetext{
${ }^{1}$ German word meaning compulsion to move, whoever is to move in that position draws or loses the chess game but would have done better if allowed to pass the move.
} 
belong to one of the following cases: i) Zero or fuzzy games, and ii) Positive or negative games where the winner has the possibility of driving the game to case (i). In addition some tempos are allowed. The main contribution consists in answering the question of how a chess player checkmates or draws knowing that he is in a winning position using combinatorial game mathematical tools. By a winning position in a game it is understood a position where one of the players makes the last move in that game and as a consequence forces his opponent to start playing in the next game and so on, until at the end plays first in the Zugzwang and draws or loses the chess game. The class of chess games considered in the paper results not to be broad enough as desired, mainly because of the restrictions imposed in its construction and the small size of the chessboard. Algebraic chess notation is used to describe the moves in the game. A basic knowledge of chess is assumed along this work. The paper is organized as follows. Section 2, begins by presenting the combinatorial game mathematical theory (CGMT) needed to understand the rest of the paper, where the classical references of Conway [1] and, Berlekamp, Conway and Guy [2] are a most, and continues by recalling Elkies' work [3] on chess positions modeling employing CGMT. Section 3, deals with the strategy plan procedure. It starts by analysing some basic blocks (games), which are defined inductively, in terms of which the chess endgame is built and proceeds explaining how all this information is compiled in order to achieve the main contribution of the paper. In section 4, some examples show how the results obtained in this work are applied. Finally, some concluding remarks are given.

\section{Preliminaries}

This section begins by presenting some combinatorial game mathematical theory (CGMT) needed (see [1] and [2]), and continues by recalling how some chess positions can be modelled employing CGMT [3].

Definition 1 If $L$ and $R$ are any two sets of games, then there is a game $G=\{L \mid R\}$.

Remark 2 All games are constructed inductively in this way and all games must eventually end.

Definition 3 The elements of $L(R)$ are called left (right) options of $G$ and the positions of $G$ are $G$ and all the positions of the options of $G$.

Let $G=\{L \mid R\}$ be a game and Left and Right two players then, given a position of $G$ player Left (Right) is restricted to move to any one of the left (right) options of $G$. The four simplest games are: $0=\{\mid\}$ (born on day 0 ) and $1=\{0 \mid\},-1=\{\mid 0\}, *=\{0 \mid 0\}$ (born on day 1) where: 
- In the game 0 , there is a winning strategy for the second player

- In the game 1 , there is a winning strategy for Left (whoever starts)

- In the game -1 , there is a winning strategy for Right (whoever starts)

- In the game $*$ there is a winning strategy for the first player to move

This motivates the following general definition.

Definition 4 Let $G$ be a game then:

- $G=0$ if there is a winning strategy for the second player

- $G>0$ if there is a winning strategy for Left (whoever starts)

- $G<0$ if there is a winning strategy for Right (whoever starts)

- $G \| 0$, called $G$ is fuzzy or confused with zero, if there is a winning strategy for the first player to move

Theorem 5 Every game $G$ belongs to one of the four outcome classes given in definition 4 .

Definition 6 Let $G=\left\{G^{L} \mid G^{R}\right\}$ be a game, the negative of $G$ denoted as $-G$ is inductively defined by the equation

$$
-G=\left\{-G^{R} \mid-G^{L}\right\}
$$

Remark 7 The negative of a zero or fuzzy game is another game of the same type.

Proceeding with the game construction algorithm given by definition 1 some more games can be defined as for example: $2=\{1 \mid\},-2=\{\mid-1\}, \uparrow=\{0 \mid *\}$, $\downarrow=\{* \mid 0\}, \Uparrow *=\{0 \mid \uparrow\}, \Downarrow *=\{\downarrow \mid 0\}$, etc.

Definition 8 Let $G=\left\{G^{L} \mid G^{R}\right\}$ and $H=\left\{H^{L} \mid H^{R}\right\}$ be two games, the sum of $G$ and $H$ denoted as $G+H$ is inductively defined by the equation

$$
G+H=\left\{G^{L}+H, G+H^{L} \mid G^{R}+H, G+H^{R}\right\}
$$

Some examples of sums of games are: $G=G+0, *+*=0, \uparrow+\downarrow=0, \uparrow+\uparrow=\uparrow$ etc.

The following two theorems play a fundamental role as will be seen in the next sections.

Theorem 9 (Copycat theorem) $G-G$ is always a zero game. 
Theorem 10 If $H$ is a zero game, then the game $G+H$ has the same outcome as $G$.

The section continues by recalling how some chess positions can be modelled employing CGMT. Let Left and Right be two players, where Left plays white and Right plays black, and consider the chess positions depicted in figure 2.1 and figure 2.2 .

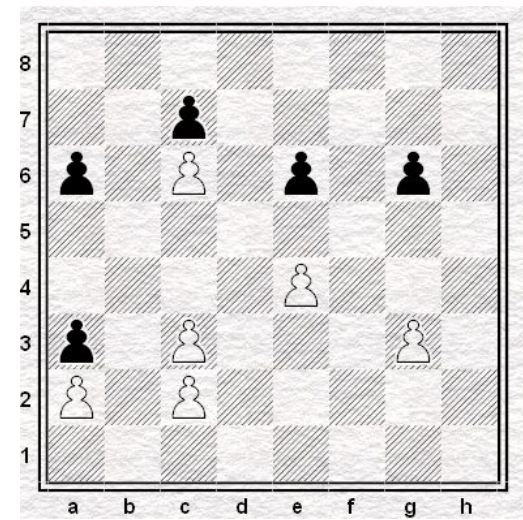

Fig 2.1

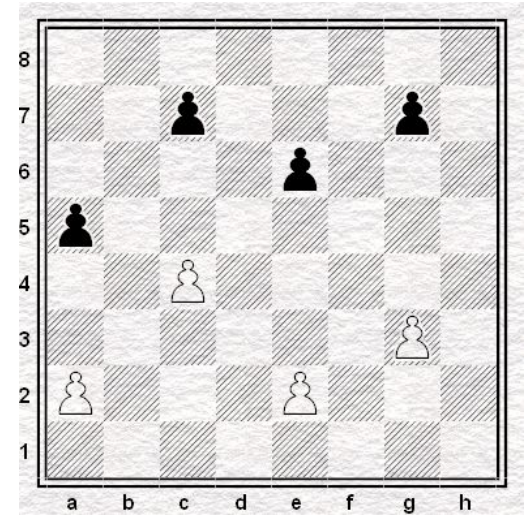

Fig 2.2

In figure 2.1, Right has two free pawn moves in column a which are modeled by the game $G=-2$, while in column c, Left has four free moves (provided the pawn in c2 moves one square at a time) therefore, $G=4$. In addition, column e, is modeled as $G=*$, while column g, as $G=0$. Figure 2.2 shows in column a (c), a positive (negative) arrow, therefore $G=\uparrow(G=\downarrow)$, and also shows in column e, the game $G=\Uparrow *$, while in column h, appears its negative $G=\Downarrow *$. The reader interested in a more detailed discussion as well as more examples is referred to [3], [4], and sections 3, 4 of this paper.

\section{Checkmate Control Procedure}

This section starts by analysing some basic independent blocks (games), which are defined inductively, in terms of which by adding them disjunctively to a Zugzwang (being careful of not altering it) the chess endgame is defined. These blocks are restricted to belong to one of the following cases: i) Zero or fuzzy games, and ii) Positive or negative games where the winner has the possibility of driving the game to case (i). In addition some tempos are allowed. The list of blocks does not pretend at any time to be exhaustive. The section, proceeds explaining how all this information is compiled in order to achieve the main 
contribution of the paper i.e., to give a strategy plan procedure to checkmate or draw.

Definition 11 Let $G$ be a chess game a winning position in $G$ is a position where one of the players makes the last move in that game and as a consequence forces his opponent to start playing in the next game and so on, until at the end plays first in the last game, the Zugzwang, and draws or loses the chess game.

Definition 12 A partial winning position in $G$ is a position where one of the players makes the last move in that game and as a consequence forces his opponent to start playing in the next game.

Let $G$ be a basic block composed of white and black pawns and its possible moves. Let $G$ be described in terms of two different color heap nim games (the moves of white and those of black) with game values the nimbers of white $* n_{w}$ and the nimbers of black $* n_{b}$ (for more on the game of nim see [1] and [2]). Let $\sim$ be a relation in the set of possible moves defined by: the nimbers of white are equal to the nimbers of black (and equal to the nimbers) $* n_{w}=* n_{b}=* n$ if and only if white move has a symmetrical black move, (which is reflexive, symmetric and transitive) then, $\sim$ defines an equivalence relation, and as a consequence an identification of all symmetrical moves is obtained,

Case i) Zero or fuzzy games:

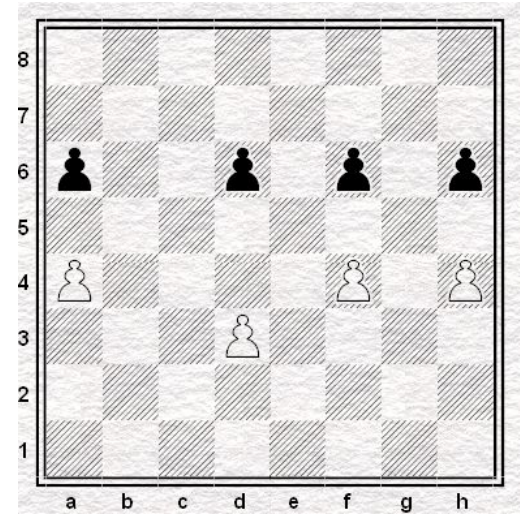

Fig 3.1

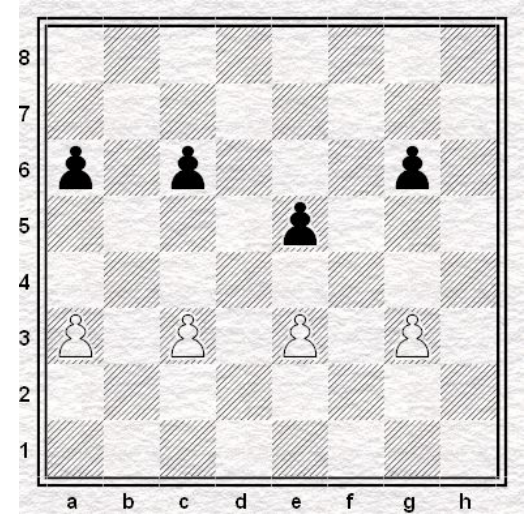

Fig 3.2

In figure 3.1, column a, shows the game $G=*$, the idea here is to move first, since whoever moves first is in a partial winning position. This game is described by a one heap nim game with one stick (the only possible symmetric 
move for both pawns) whoever takes first the stick wins. Column d, defines the game $G=0$ where the player who plays second is in a partial winning position. Taking advantage of the symmetry that the movements of the game has above and bellow the line that divides row 4 from row 5 (one symmetrical move) and making the necessary identification, $G$ can be described as a nim game composed of two heaps of size one $G=* 1+* 1=* 0=0$ and therefore once one of the players moves first the other player simply applies the Copycat theorem (theorem 9), forcing his opponent to start playing in the next game (no sticks left).

Remark 13 Notice that the main goal for each one of the players is to drive the game to a zero game where his adversary loses it.

Columns $\mathrm{f}$ and $\mathrm{h}$, show an overall game $G=*+*=0$ which again is equivalent to a nim game composed of two heaps of size one where the Copycat theorem applies and tells the winner player how to beat his opponent.

In figure 3.2, columns a and c, show the game $G=0+0=0$ which is equivalent to a nim game composed of two heaps of size two where the Copycat theorem applies in column a, b, or mixing both (since all of them belong to the same subset of the partition). Columns e and $g$, show the game $G=*+0=*$ which after the first move, reduces to the zero games already analysed in figure 3.1 namely, column $\mathrm{d}$, or columns $\mathrm{f}$ and $\mathrm{h}$.

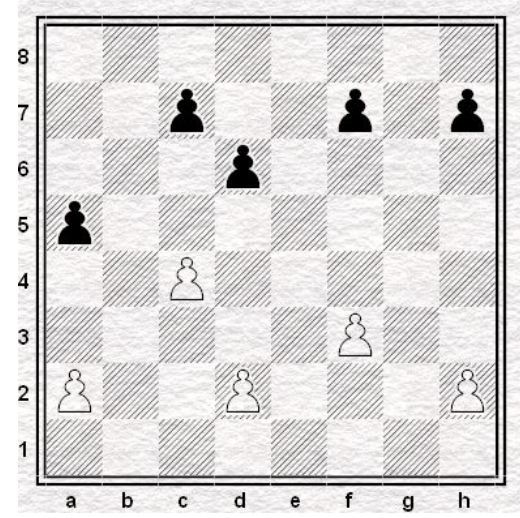

Fig 3.3

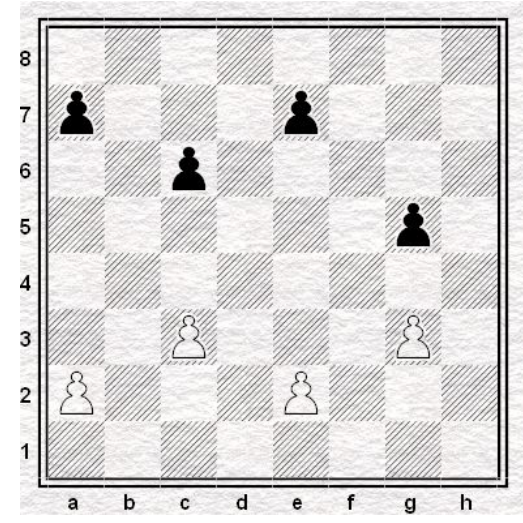

Fig 3.4

In figure 3.3, columns a and c, show the game $G=\uparrow+\downarrow=0$ with symmetric moves (for each move white (black) makes in column a, there is a copy for the opposite player in column c and viceversa) therefore, it is equivalent to a nim game composed of two heaps of size two with the possibility, that the players 
have, of removing two sticks at a time. Therefore, this subcase is handled with the Copycat theorem. Columns d an $\mathrm{f}$, show the game $G=\Uparrow *+\Downarrow *=0$ whose analysis follows the same lines done in the previous subcase. Finally, column h, shows a game $G=0$ equivalent to a nim game composed of two heaps of size two with the possibility, that the players have, of removing two sticks at a time. This subcase is handled with the Copycat theorem.

In figure 3.4, columns a and c, show the game $G=0+0$ which is handled by applying the Copycat theorem in the same column in order to preserve the symmetry of the movements i.e., each column belongs to a different subset of the induced partition. The game $G$ is equivalent to two disjoint (two heaps) nim games, one with two sticks (with the possibility of removing two sticks at a time) and the other with one. Columns e and g, show a game with value $G=0+*=*$ therefore the first player to move forces his opponent to zero by reducing the game to the subcase of column $\mathrm{h}$ in figure 3.3.

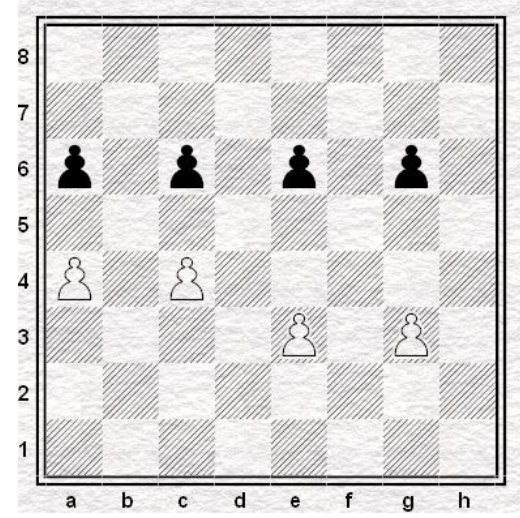

Fig 3.5

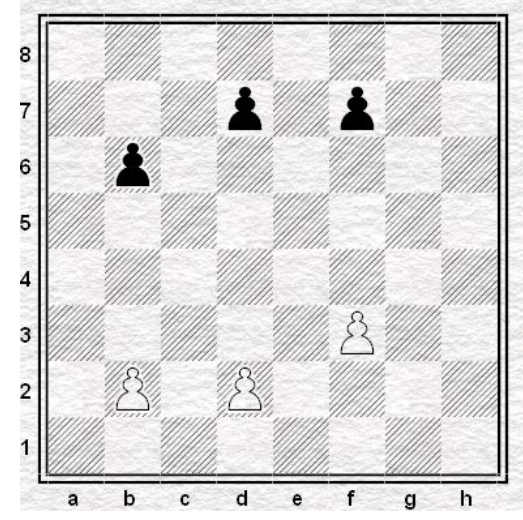

Fig 3.6

In figure 3.5, columns a, c, e and $\mathrm{g}$, show the game $G=*+*+0+0=0$ whose analysis follows from the Copycat theorem applied to any mixture of the columns, since all possible movements belong to the same subset of the partition.

In figure 3.6, columns $\mathrm{b}, \mathrm{d}$ and $\mathrm{f}$, show the game $G=\Uparrow *+0+\Downarrow *=0$ which is equivalent to two disjoint (two heaps) nim games one with three sticks and the other with two (both of them with the possibility of removing two sticks at a time) its analysis follows by applying the Copycat theorem in columns b and $\mathrm{f}$ and in column $\mathrm{d}$ in order to preserve the symmetry i.e., the identification. 


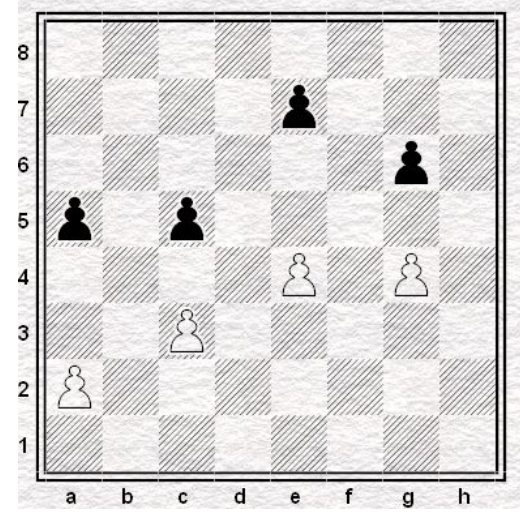

Fig 3.7

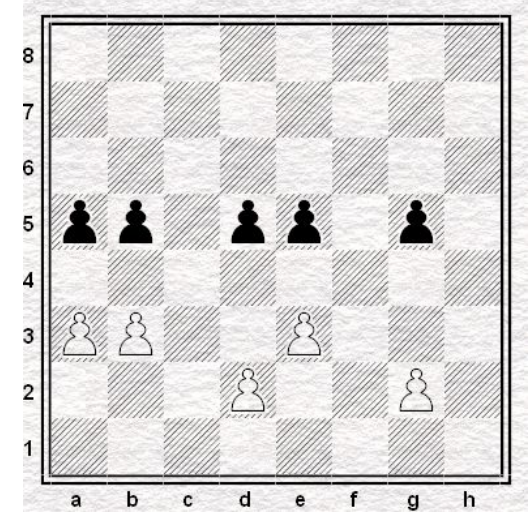

Fig 3.8

In figure 3.7, columns a and c, show the game $G=(\uparrow *) \| 0$ therefore the player who moves first is in a partial winning position. If white starts, by moving his pawn from a2 to a3, he succeeds to reduce this game to the zero game shown in columns $\mathrm{f}$ and $\mathrm{h}$ of figure 3.1. However if black starts he can do the same, moving his pawn from a5 to a4, restricting the double move of the white pawn in a2 to a single one. In either case the first player is in a partial winning position. Columns e and g, show the game $G=(\downarrow *) \| 0$ whose analysis is similar to the previous one made.

In figure 3.8, columns a and $\mathrm{b}$, show the game $G=0$ where whoever moves first is forced to play in the next game without having to trade pawns. The analysis is as follows: If white moves his pawn from a3 to a4, black moves from b5 to b4, forcing white to play in the next game, the same idea applies to the rest of the possible movements in this game.

ii) Positive or negative games where the winner has the possibility of driving the game to case (i).

Remark 14 Notice that it will always be possible for the winner to drive the game to case (i), because of the inductive nature of definition (1) where the basis is the game $G=\{\mid\}=0$. 


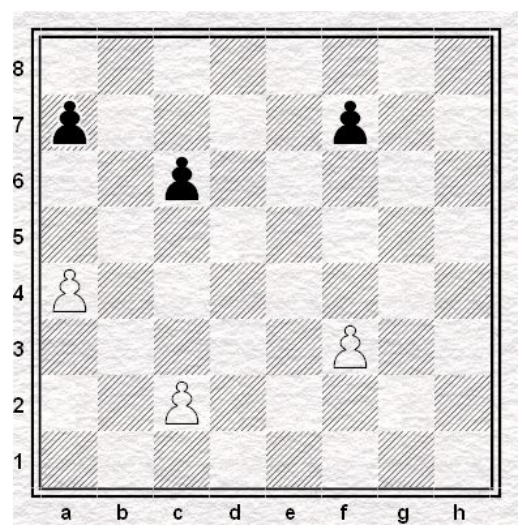

Fig 3.9

In figure 3.8, columns $\mathrm{d}$ and e, show the positive game $G=\uparrow$ where white always is in a partial winning position. The possibilities are: If white starts then, moving his pawn from $\mathrm{d} 2$ to $\mathrm{d} 3$ reduces the game to the previous game $G=0$ (columns a and b) and he is done. If black starts, no matter what moves he makes (pawn d5 to $\mathrm{d} 4$, forcing a double trade, finishing with the white pawn $\mathrm{d} 2$ in $\mathrm{d} 3$ blocking the black pawn in $\mathrm{d} 4$ or, e 5 to e 4 for which the pawn in $\mathrm{d} 2$ uses his double move to $\mathrm{d} 4$ blocking all possible moves) white is always in a partial winning position forcing black to play in the next game. Column g, shows the positive game $G=\uparrow$ where white is always in a partial winning position. The analysis is as follows: If white starts he simply uses is double move to force black to play in the next game, while if black starts, he reduces the game to $G=*$ (which was already analysed, see figure 3.1 column a). In the end black is forced to play in the next game. In figure 3.9, column a, shows the negative game $G=\downarrow$ whose analysis follows the same lines of it previous positive case. Column c, shows the positive game $G=\Uparrow *$ where if white starts he moves one step to reduce the game to $G=0$ while if black starts, he reduces the game to the positive game $G=\uparrow$ (already analysed, see figure 3.8 column g). Therefore in either case white is in a partial winning position. Column $\mathrm{f}$, shows the negative game $G=\Downarrow *$ whose analysis follows the same lines of its previous positive case.

Some tempos are next treated. Figure 3.10, column a, pawns a3 and a6 show the game $G=*$ (already analysed, figure 3.1 column c) however, if white starts he loses because of the one tempo black has with his a7 pawn. Column $\mathrm{c}$ is the reflected version of the previous one. In column e, pawns e2 and e6 show the game $G=\Uparrow *$ (already analysed, see figure 3.9 column c) however white loses because of the one tempo black has with his e7 pawn. Column $g$ is its reflected version. 


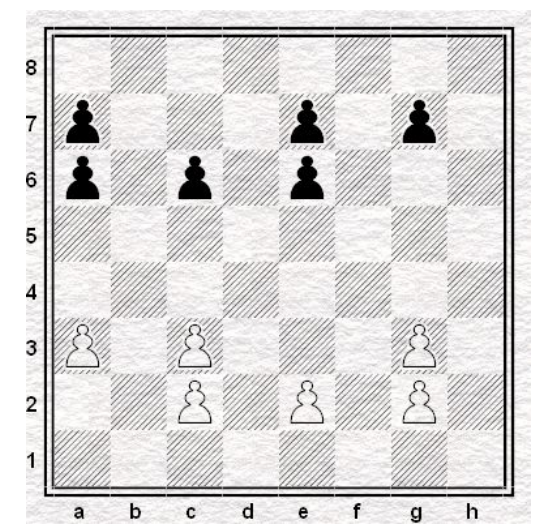

Fig 3.10

Remark 15 As was said at the beginning of this section the list of basic blocks proposed never tried to be exhaustive and some other possibilities of interest can be proposed.

Once the basic blocks have been introduced and adding them disjunctively to a Zugzwang (being careful of not altering it) the chess game $G$ is built. The chess game is expressed as a disjunctive sum of subgames $G=G_{1}+G_{2}+\ldots+G_{n}$ where knowing that eventually all the basic blocks added will finish in a blocked configuration one concludes that whoever plays first in the Zugzwang draws or loses the game. Even more, since the Zugzwang game is considered to have a zero value (whoever is to move in the Zugzwang draws or loses the chess game but would have done better if allowed to pass the move), from theorem 10 all what matters is to know what happens in the basic blocks. Therefore, computing the value of $G$ reduces to computing the value of the sum of the basic blocks where depending of its sign, from the analysis made for each one of the blocks' cases, a strategy plan procedure to checkmate or draw, which depends on the chess game built, can be proposed. This will be illustrated by means of some examples in the next section.

\section{Applications}

This section illustrates how all the analysis made in the previous section for several building blocks together with the possibility of decomposing the chess game as a disjunctive sum of subgames, one of them being the Zugzwang, and theorem 10, is applied in order to get a strategy plan procedure to checkmate or draw. Some more interesting examples can be proposed, these are left to the imagination of the reader. 


\subsection{Example 1}

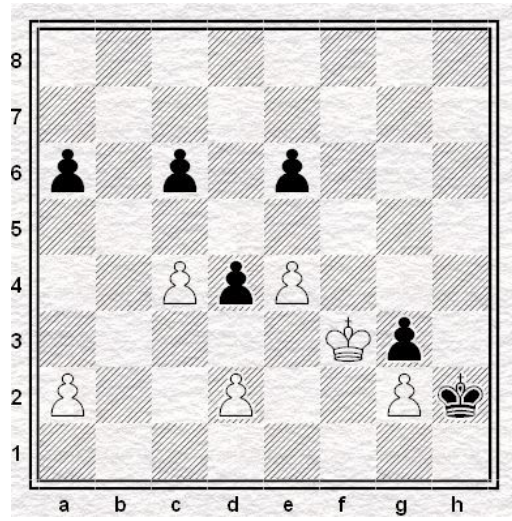

Fig 4.1

Strategy Plan Procedure Analysis: This is a positive game composed of three (*) blocks, and one $(\Uparrow *)$ block, $G=\Uparrow *+*+*+*=\Uparrow>0$.

If black starts:

- Case 1. Black reduces any $(*)$ block to the $(0)$ block which results in $G=\Uparrow *+*+*=\Uparrow *>0$ then, white reduces $(\Uparrow *)$ to zero by moving his pawn from a 2 to a3 and gets two blocks equal to $(*)$ (see figure 3.1 columns $\mathrm{f}$ and $\mathrm{h}$ and its analysis) and one equal to zero (see figure 3.1 column $\mathrm{d}$ and its analysis) where playing copycat in each block, white is in a winning position.

- Case 2. Black moves his pawn from a6 to a5 which results in $G=\uparrow$ $+*+*+*=(\uparrow *) \| 0$ but white is the first one to play, then he moves his pawn from a2 to a4 getting a game $G=*+*+*+*$ i.e four $(*)$ blocks or to two $(*)$ blocks therefore, by the same token as before white plays copycat and forces black to be the first to move in the Zugzwang.

If white starts:

White reduces the $(\Uparrow *)$ block to the $(*)$ block which results in the game $G=*+*+*+*=0$ where as in blacks case 2 , white plays copycat and therefore, white is in a winning position.

Remark 16 Notice that by adding an extra black pawn, as for example one in c7, black is now the one who is in a winning position since using this tempo at the end (when all pawns get blocked) he forces white to move first in the Zugzwang (see the tempos presented in figure 3.10 and its analysis). 


\subsection{Example 2}

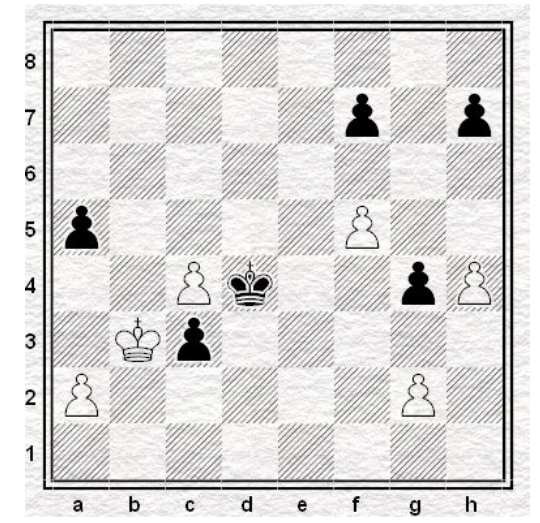

Fig 4.2

Strategy Plan Procedure Analysis: This is a zero game composed of two arrow blocks (one positive and one negative), and two (*) blocks, $G=\uparrow+*+*+\downarrow=0$. As a consequence the player who plays in second is in a winning position by playing copycat (see figure 3.1 columns $\mathrm{f}$ and $\mathrm{h}$, and figure 3.3 columns a and c, plus their respective analysis).

\subsection{Example 3}

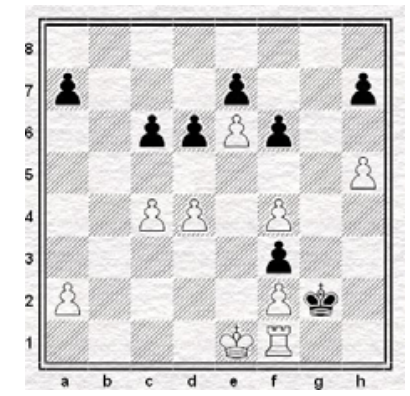

Fig 4.3

Strategy Plan Procedure Analysis: This is a game composed of two zero blocks (see figure 3.4 column a, figure 3.8 columns a, b, and its analysis), and two $(*$ ) blocks (see figure 3.1 columns $\mathrm{f}$ and $\mathrm{h}$, and its analysis). Therefore, since $G$ is equal to zero $(G=0+0+*+*=0)$, the first player to move is the one who plays first in the Zugzwang. The strategy plan procedure consists in playing copycat in the two $(*)$ blocks, playing copycat in the zero block (column a), and forcing a zero in the block given by columns $\mathrm{c}$ and $\mathrm{d}$. 


\section{Conclusions}

This work gives a partial answer to one of the most important questions in chess, how a chess player checkmates or draws knowing that he is in a winning position. This is achieved using combinatorial game mathematical theory. The strategic planning procedure can be of some utility for teaching beginner players basic facts about some endgames.

\section{References}

[1] J. H. Conway, On Numbers and Games, A K Peters, 2001.

[2] E. Berlekamp, J. H. Conway, R. Guy, Winning Ways For Your Mathematical Plays, I: Games in General, Academic Press, 1976.

[3] N. D. Elkies, "On Numbers and Endgames: Combinatorial Game Theory in Chess Endgames", Games of No Chance MSRI Publications. Volume 29, 1996.

[4] N. D. Elkies, "Higher Nimbers in Pawn Endgames on Large Chessboards", More Games of No Chance MSRI Publications, Volume 42, 2002.

Received: June 10, 2007 